\title{
Ubiquitous Infrastructure and Smart Service on City Gas Environments in Korea
}

\author{
Jeong Seok Oh, Jang Sik Park, Jeong Rock Kwon \\ Appliances Research Department, Safety Research Division \\ Institute of Gas Safety R\&D, Korea Gas Corporation \\ Siheung Si, Gyeonggi-Do, Korea \\ dbstar@kgs.or.kr
}

\begin{abstract}
The information technology paradigm shifts to smart service environment, as ubiquitous technologies are used in the latest industry trend. The major features of ubiquitous smart service are high dynamism and heterogeneity of their environment and the need for context awareness. In order to resolve these features, it is necessary to develop middleware that meet various new requirements. This paper designed middleware on ubiquitous smart service for enhancing the safety and reliability to city gas environment in Korea. The object of this paper will support cornerstone in order to construct the framework of intelligent infrastructure and service for autonomic management.
\end{abstract}

Keywords-Infrasturcture; smart service; middleware

\section{INTRODUCTION}

The information technology paradigm shifts to smart service environment, as ubiquitous technologies are used in the latest industry trend. Ubiquitous technologies are capable of innovating public services, increasing the quality of life, and enhancing business productivity as constructing intelligent network[15]. However, it is necessary to develop network infrastructure based on ubiquitous technologies on considering particularly industry characteristics and smart service platform by analyzing the collected context-aware information in order to apply those technologies[14, 2, 1, 3].

Although a few industries might be introduced ubiquitous technologies, there are a little convergence cases between ubiquitous and gas fields such as GASNET(Gasline Network Sensor System)[11]. GASNET is developed by NETL(National Energy Technology Laboratory). This system could provide the increased understanding of distribution network, leading to cost benefits estimated to be over $\$ 10$ million annually, nationwide. However, this system is only applicable in natural gasline on USA. Furthermore, applying ubiquitous technologies to specific industry facilities must design the applicable infrastructure through analyzing characteristics of the surrounding environment, and require essentially smart service architecture for enhancing the safety and reliability.

Ubiquitous smart services might rely on middleware platform $[13,10,9]$. In general, middleware platforms manage interaction between application layer and distributed sub network layer. The ubiquitous middleware platforms must need various requirements such as access mechanism, control mechanism, abstraction to heterogeneous sub network, the high level modeling, and management of meat information and context awareness.

This paper is to design ubiquitous infrastructures and smart service platform for enhancing the safety and reliability to city gas facilities in Korea. Firstly, we explain designing ubiquitous infrastructures from various different networks which can be applicable on city gas environments in Korea. So, we select four target city gas facilities, and then analyze characteristics of target environment and measurable parameters. Moreover, we make prototype devices per target facility using applicable network method, and then derive the applicable design criteria on the city gas facilities of major cities in Korea. Secondly, we explain the outline of ubiquitous smart service to city gas environments, and show two application layers of smart service. Furthermore, we define requirements for middleware platform to city gas facilities, and show three middleware layers for smart city gas management. The object of this paper will support cornerstone in order to construct the framework of intelligent infrastructure and service for autonomic managing related facilities in Korea.

\section{RELATED WORKS}

[13] proposed a few requirements for a ubiquitous context awareness middleware. In this study, ubiquitous context awareness is the capability of middleware to provide access to heterogeneous, distributed, and un-anticipated context information. This research suggested twelve requirements for middleware about ubiquitous context awareness. Twelve requirements are distributed context management, support for context evolution, dynamic context discovery, scope of context perception, multiple mechanisms and policies for accessing context, extensible abstraction for accessing and using context, management of application dynamic loading, abstract handling of context interest, architectural independence, decoupling between context management and inference mechanisms, easy incremental deployment/distributed administration and standardization, suitable programming tools for context discovery.

[10] proposed OpenCOPI(Open Context Platform Integration). This platform integrates several context middleware in order to provide a unified context service for 
ubiquitous application. Moreover, this encapsulates the underlying middleware platforms and then represents platforms as services. As the use of platform simplifies the development of ubiquitous applications, it takes advantage of the specific features of individual middleware, abstracting away their differences for the application. Therefore, the platform allows the integration of different context middleware by using an approach of automatic service composition. Also, OpenCOPI adopted the CONON ontology that is composed of two ontology layers. The first layer captures general concepts about ubiquitous environment, comprising only features of basic contextual data. The second layer is made up of domain specific ontology. The domain specific ontology defines the details of general concepts each sub domain.

[9] were focused on architecture for mobile network supported wireless sensor network. Designing the architecture utilizes the existing infrastructure to interconnect independent wireless sensor network. The middleware layer is called M2M system in this study. The M2M system can analyze high level application requests and identifies the information that receives from the sensors. In other words, the system aggregates information from all small information providers and provides higher level services to end users. The main components of M2M system are service control function, request analyzer, and service registry.

[5] proposed MiLAN(Middleware Linking Applications and Network). MiLAN allows sensor network applications to specify their quality needs and adjusts the network characteristics to increase application lifetime while still meeting those quality needs. The middleware receives from the individual applications about their QoS requirements over time and how to meet these QoS requirements using different combinations of sensors, the overall system about the relative importance of the different applications, and the network about available sensors and resources such as sensor energy and channel bandwidth. Also, this research explained benefit from a middleware that proactively affects different characteristics of network and the design feature of the middleware such as inherent distribution, dynamic availability of data sources, resource limitation, and cooperative applications.

\section{UBIQUITOUS INFRASTRUCTURE ON CITY-GAS IN KOREA}

Fig. 1 shows ubiquitous smart infrastructure and service in our research scope. We select four principal target facilities in Korea. Also we define stress/vibration in above pipelines, leakage gas/current/water level/pressure in gas valve boxes, and corrosion in the test boxes for underground pipeline as parameters of context awareness in order to develop ubiquitous wireless infrastructures.

In order to send measurable data in city gas facilities, various methods may be used. Although the existing methods used wired communication such as PLC and Internet, wireless communication methods are introduced with a few benefits. Actually, communication devices are already installed in a few city gas facilities like as gas governor on the major city environment in Korea. These facilities are generally set up per $1 \mathrm{~km}$ distance length on city gas environment [12]. Therefore, we are to apply near distance network technologies like as sensor network if the distance between target facility and gas governor is less than $1 \mathrm{~km}$. On the other hand, we are to use long distance network technologies like as mobile commercial network [8].

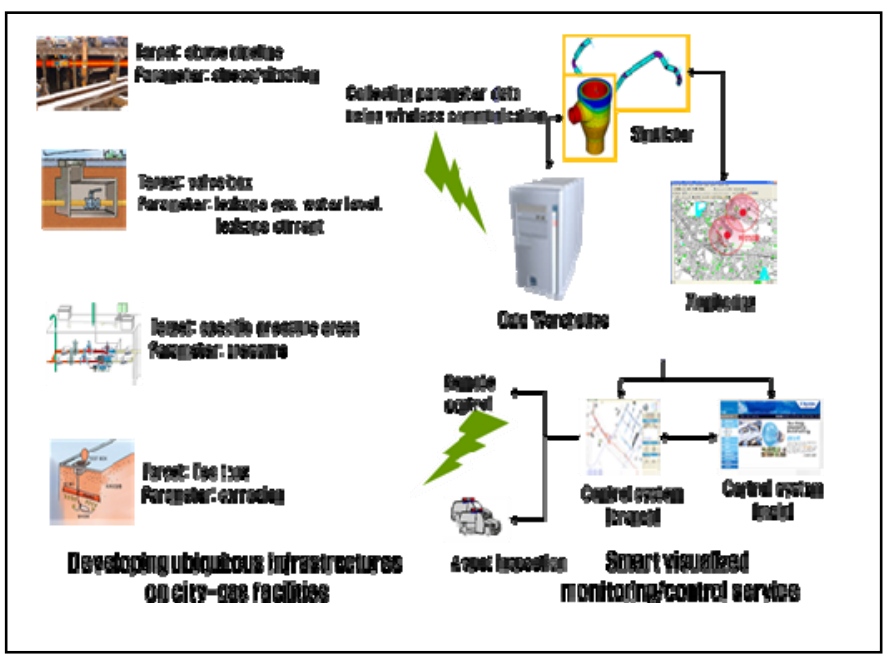

Figure 1. Ubiquitous smart infrastructure in city-gas environment

Fig. 2 shows our idea of data transmission for using the major city environment in Korea. As gas governors are generally use wired communication and set up per $1 \mathrm{~km}$, they might be played a pivotal role for developing wireless city gas infrastructures. If target facilities exist within $1 \mathrm{~km}$ of gas governor, we are to develop sensor network with less than $900 \mathrm{MHz}$ bandwidth or $2.4 \mathrm{~Hz}$ bandwidth $[6,16]$. According to our experimental tests, $2.4 \mathrm{HZ}$ bandwidth is better than in open ground environment on the major city of Korea. In addition, Zigbee might show longer transmission distance, lower transmission output power, and self-organization network.

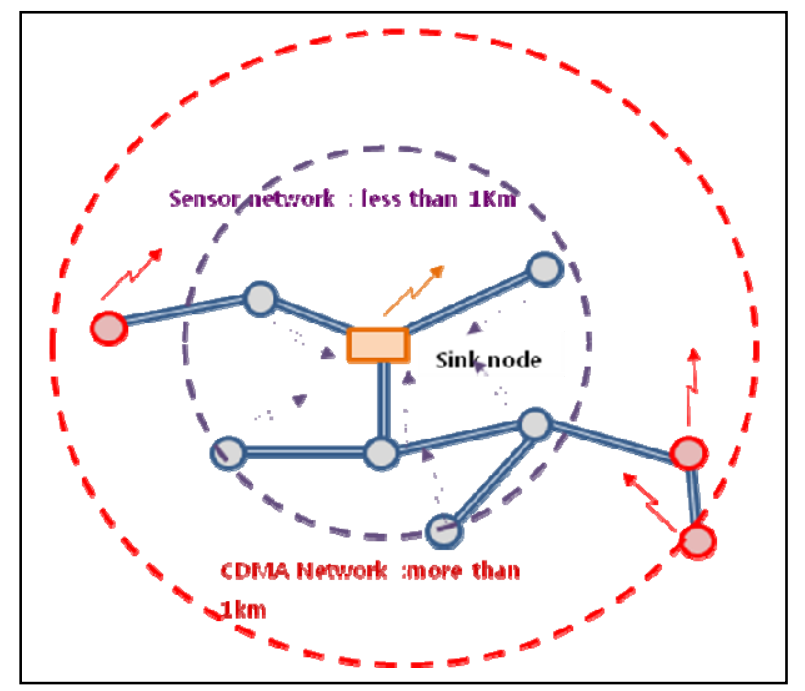

Figure 2. The idea of data transmission for city gas environment in Korea

However, Zigbee is not applicable in the half-closed underground environments because $2.4 \mathrm{GHz}$ bandwidth did not get through concrete or steel environment. The bandwidth less than about $900 \mathrm{MHz}$ might be preferable to the half-closed underground environments. The transmission from closed to 
open environment simultaneously utilized Zigbee and $424.7 \mathrm{MHz}$ on city gas environment in our research because the maximum distance is more than 80 meters. 80 meters might mean minimum distance from the closed underground to the nearest structures in order to be feasible wireless network on the major metros environment in Korea.

Table 1 shows the distance between target facility and gas governor in Korea. Therefore, test boxes for underground pipeline, the third-party damage pipeline and valve boxes might be able to apply CDMA or sensor network (ZigBee + $424.7 \mathrm{MHz}$ ) based on the relative location of gas governor in city gas environment. The above pipeline might make use of CDMA.

TABLE I. DISTANCE BETWEEN TARGET AND GAS GOVERNOR

\begin{tabular}{|l|c|}
\hline \multicolumn{1}{|c|}{ Target } & Distance \\
\hline Test boxes for underground pipeliens & $1 \mathrm{~km} \leq$ or $1 \mathrm{~km} \geq$ \\
\hline Valve boxes & $1 \mathrm{~km} \leq$ or $1 \mathrm{~km} \geq$ \\
\hline The $3^{\text {rd }}$-party damage pipeline & $1 \mathrm{~km} \leq$ or $1 \mathrm{~km} \geq$ \\
\hline Above pipeline & $1 \mathrm{~km} \geq$ \\
\hline
\end{tabular}

Fig. 3 shows test-bed for test boxes and valve boxes for experimenting wireless near distance network. This test-bed is capable of monitoring and controlling corrosion, leakages of gas, water level, leakage of current, pressure. Also, other parameters are measured for managing facility information such as status, history, and so on. The test-bed consists of ten monitoring points and three gas governor rooms, including the closed environment. The distance among monitoring points would be appointed as the maximum of 495 meters and minimum of 43 meters.

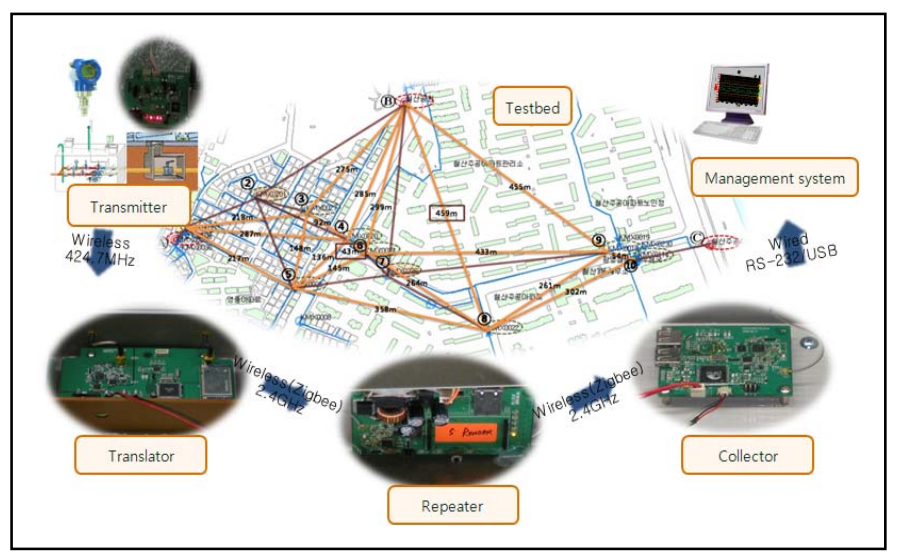

Figure 3. Test-bed for sensor network

Our network components are composed of transmitter, translator, repeater and collector as wireless two-tier network. The transmitter sense values of measurable parameter and transmit to translator using $424.7 \mathrm{MHz}$ bandwidth. The translator changes $424.7 \mathrm{MHz}$ into $2.4 \mathrm{GHz}$ (ZigBee), and retransmits data to other ZigBee components. This unit participates in mesh network of IEEE 802.15.4a and includes all repeater functions. The repeater receives data from another translator and repeater, and transmits data to the collector. This unit support only IEEE 802.15.4a wireless network, and also participate in mesh network. The collector transmits the received data from repeater to management system using internet. This unit plays a key role as network coordinator of whole IEEE 802.15.4a network, and controls data transmission/reception and organization of mesh network. The translator and repeater request addition of mesh network to other component of network when booting firstly, and then the collector reorganizes mesh network.

Fig. 4 shows test-bed for above pipelines under bridges in order to test wireless long distance network. This test-bed consists of eight above pipelines on bridges of Gyeonggi-do in Korea. The measured parameters are stress, vibration, identification, and so on. We develop prototype devices in order to obtain values of parameter and transmit those values using CDMA. These prototypes are roughly divided into the stress module, the power module, the vibration module, the transmission module and main unit. Especially, the stress and vibration module can extend five channels, respectively.

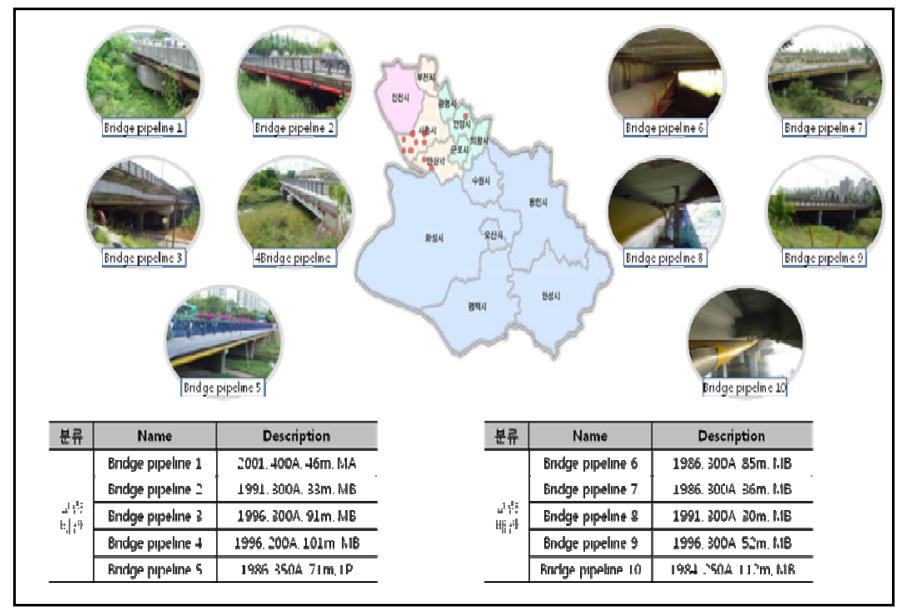

Figure 4. Test-bed for CDMA

\section{SMART City Gas PlatForm}

Furthermore, our research aims to evolve smart visualized monitoring and control service. We are to provide autonomic processes such as monitoring risk parameters, accumulating information, controlling target facilities, and displaying visualized analysis results in these services []. Therefore, middleware is necessary for integrating information and consistent service to achieve the ubiquitous smart service.

The outline of smart service for city-gas facilities is shown as Fig. 5. Sensing data of each network are translated by the private adaptor, incorporated into common message, and then transmitted to middleware through common network interface. The adaptor is assigned per sub network layer, and plays an import role in order to have not influence on overall system, whenever addition or drop specific sub network infrastructure. A middleware manage event processing, autonomic reporting, information processing, and so on. Also, a middleware can connect other application systems, as it accesses to application service through standard open interface. 


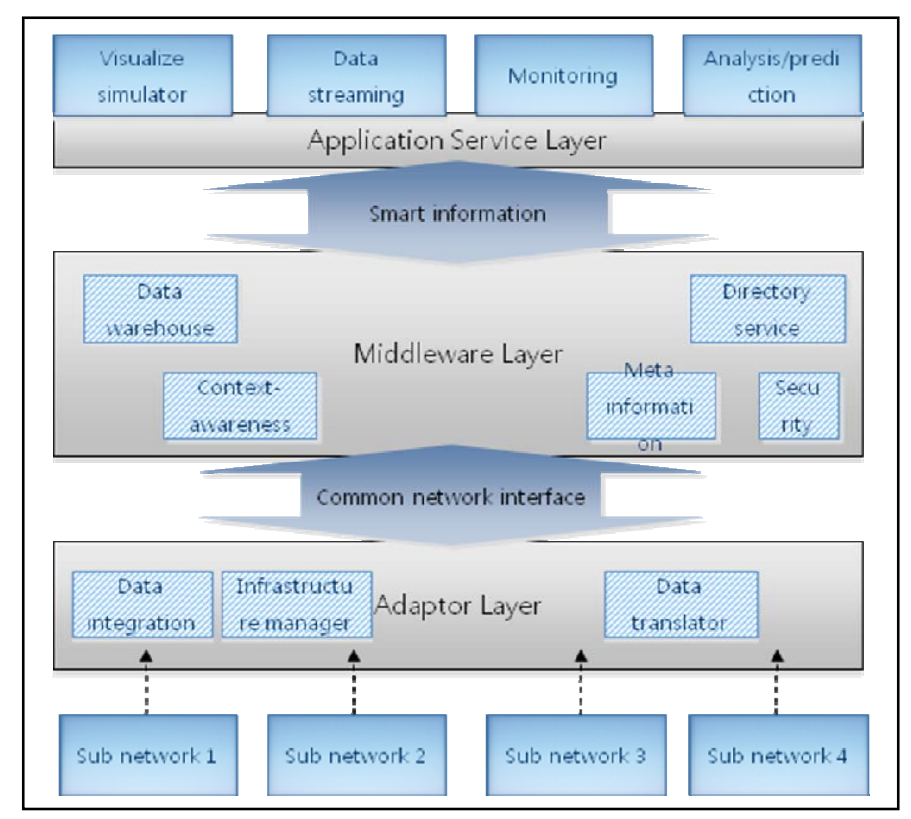

Figure 5. Example of a figure caption. (figure caption)

The adaptor layer should be interacted each individual adaptor for heterogeneous sub network. Moreover, adaptor communicates the sensing data model component for taking the related information and format. For instance, the sensing data model component can use XML in order to provide the united message data type using template form. The adaptor might consist of reception part, analysis part, translation part, transmission part. The reception part receives data through various sub network interface. The analysis part verifies data integrity. The translation part modifies the analyzed data into the united message data with referencing profile information of the sensing data model component. The data transmission part sends data to middleware or receives data from middleware.

The middleware layer must store all related information to data warehouse, manage all information, and evolve intellectualization using data mining methodologies. Using data mining methodologies is able to display autonomic risk analysis and prediction. In our case, we improve the fuzzy-k$\mathrm{NN}$ algorithm. Our algorithm keeps the class membership information and differs from processing the initial membership in order to provide practical data mining algorithm.

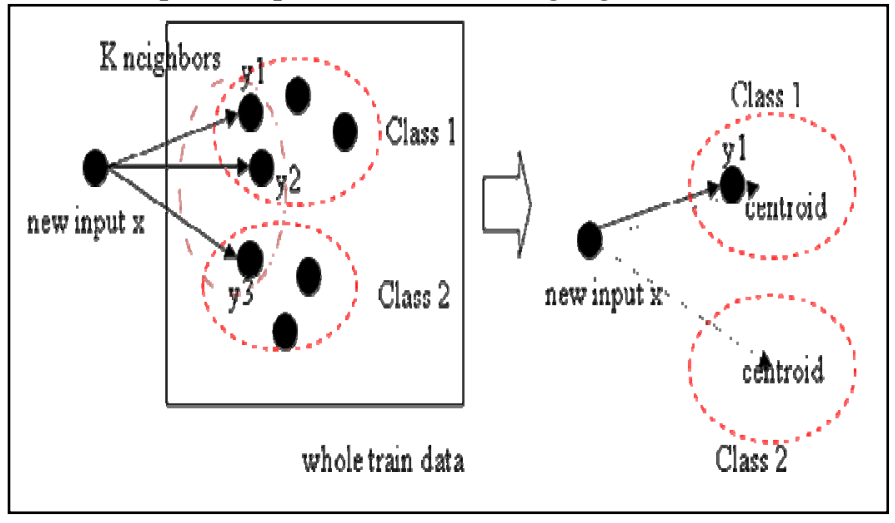

Figure 6. Example of a figure caption. (figure caption)
Fig. 6 shows our idea for information processing. We consider how each class is similar to $\mathrm{x}$ for calculating the initial membership value. In our method, initial membership between $\mathrm{x}$ and $\mathrm{y} 1$ is defined by $\mathrm{y} 1$ 's class information and distance between $\mathrm{x}$ and midpoint of each class.

Another function of the middleware layer can deal with interaction between middleware and services by means of supplying the standard open application interface. Especially, meta-data and directory service are able to provide efficient information detection. The detailed design of directory service component shows Fig. 7. ID manager processes creation, grant, and manage identifier. DAO provides connection of database. The location manager finds location of meta-data. Others carry out registration, deletion, inquiry, modification, and update to meta-data, respectively.

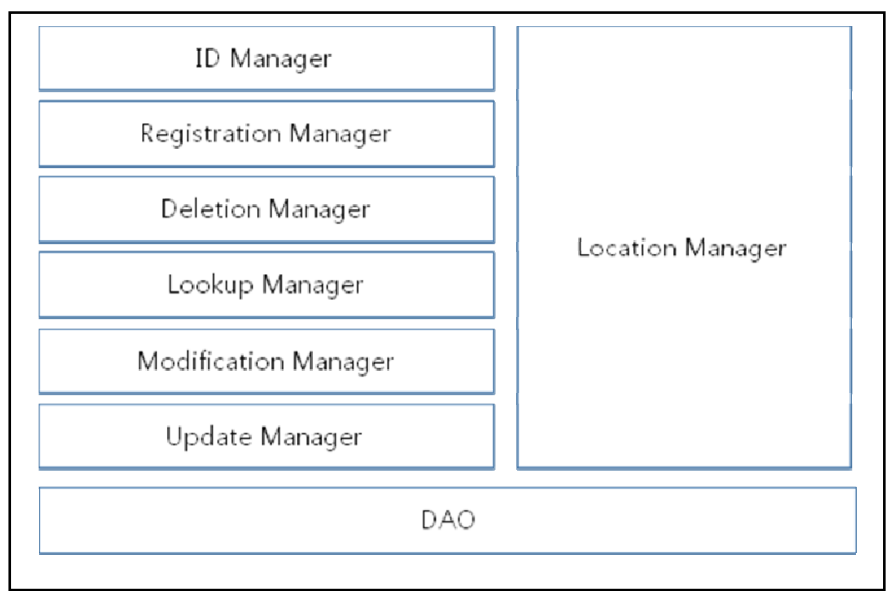

Figure 7. Example of a figure caption. (figure caption)

The application service layer processes user requirements and provides efficient results. The visualized simulator imagines point of leakage gas on pipeline according to simulation using cross correlation method. The data streaming component carries out sending separately in case of large data size and processing efficiently by data queuing. Also, web based monitoring service provide user interface and integrated other service component such as analysis and prediction as shown Fig. 8.

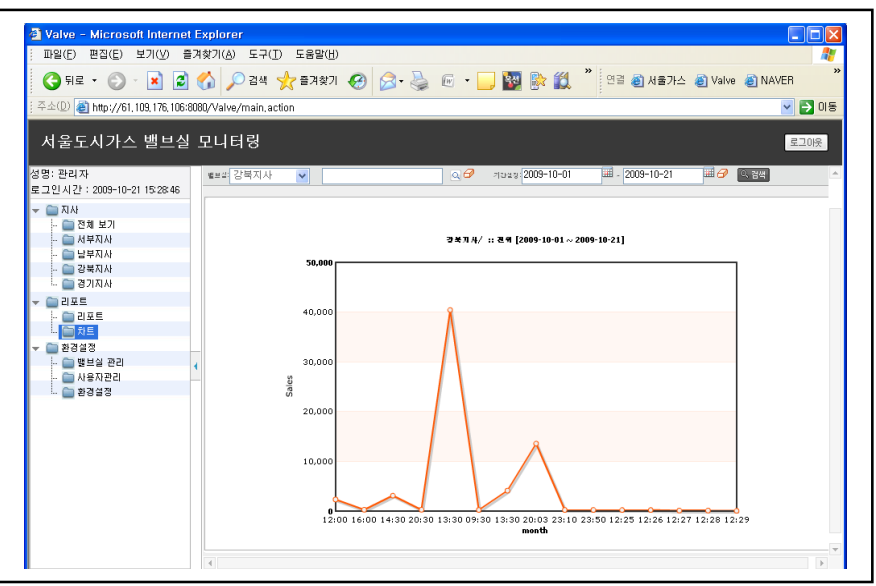

Figure 8. Example of a figure caption. (figure caption) 


\section{CONCLUSIONS}

Applying ubiquitous technologies to specific industry facilities must design the applicable infrastructure through analyzing characteristics of the surrounding environment, and require essentially smart service architecture for enhancing the safety and reliability. A smart service is the high dynamism and heterogeneity of their environment. To cope with these features, ubiquitous smart services might rely on middleware platforms. In general, middleware platforms manage interaction application layer and sub network layer. Moreover, middleware platforms for ubiquitous must need various requirements such as access mechanism, control mechanism, abstraction to heterogeneous sub network, the high level modeling, and management of meta information and context awareness.

This paper designed ubiquitous infrastructures and smart service platform for enhancing the safety and reliability to city gas facilities in Korea. Firstly, our paper might design ubiquitous infrastructures which can be applicable from different various networks on city gas environment in Korea. So, we collect stress/vibration in above pipeline, leakage gas/water level/leakage current/pressure in valve boxes, and corrosion in test boxes. Because city gas facilities with communication device are generally set up per $1 \mathrm{~km}$ distance on major city environment in Korea, we used sensor network or commercial network depending on distance between target and gas governor. In addition, the transmission from closed to open used simultaneously $2.4 \mathrm{GHz}$ and $424.7 \mathrm{MHz}$ in order to meet requirements. Furthermore, we made prototype devices per target facility using applicable network method, constructed test-beds, and then derived the applicable design criteria on the major city environment in Korea.

Next, our paper might design the outline of smart service on city gas environments. This service architecture plays an important role in order to have not influence on overall system whenever addition or drop sub network, manages all related data as the meaning information, and processes efficiently requests or results. This architecture is divided by the adaptor layer, middleware layer and application service layer. The adaptor layer provides independency to various sub heterogeneous network by providing consistent common interface. The middleware layer manages all information and evolves intellectualization using data mining. Also, this layer deals with interaction between middleware layer and service layer by means of supplying the open interface. We developed the intellectualized algorithm using $\mathrm{k}-\mathrm{NN}$, and provided efficient detection method using meta-data and directory service. The application service layer processes user requirements and provides efficient results. The object of this paper will support cornerstone in order to construct the framework of smart service infrastructure for autonomic management on city-gas facilities in Korea.

\section{ACKNOWLEDGMENT}

This work was supported by MIKE(Ministry of Knowledge Economy) under the program of ETI(Energy Technology Innovation). This paper is a result of "Research Group of Energy Safety for Next Generation". (Project No 2007-MCC23-P-03-1-000).

\section{REFERENCES}

[1] F. Akyidiz, W. Su, and Y. Sankara, "A Survey on Sensor Network" Journal of IEEE Communication Magazine, vol. 40, no. 8, pp. 102-114, 2002

[2] D. Culler and W. Hong, "Wireless Sensor Network", Journal of ACM Communication, vol. 47, no.6, pp. 30-33, 2004.

[3] A. Ganek and T. Corbi, "The Dawning of the Autonomic Computing Era", Journal of IBM system, vol. 42, No. 1, pp. 5-18, 2003.

[4] J. Han and Y. K. Kim, "A Fuzzy k-NN Algorithm using Weights from Variance of Membership Values”, IEEE CVPR Conference, pp. 394-399, 1999

[5] W. B. Heinzelman, A. L. Murphy, S. H. Carvalho, and M. A. Perillo, "Middleware to Support Sensor Network Applications", Journal of IEEE Network, vol. 18, No. 1, pp. 6-14, 2004.

[6] G. Held, "Data Over Wireless Networks Bluetooth, WAP, and Wireless LANs", McGraw-Hil, 2001.

[7] IEEE 802.15.4 TM, "MAC and PHY Specification for Low-Rate Wireless Personal Area Networks", IEEE Computer Society, 2003.

[8] R. S. Koodli and C. E. Perkins, "Mobile Inter-Networking with IPv6: Concepts, Principles, and Practives", Wiley-Interscience, 2007.

[9] S. Krco, V. Tsiatsis, and K. Matusikova, "Mobile Network Supported Wireless Sensor Network Services", the IEEE International Conference on Mobile Adhoc and Sensor System”, pp. 1-3, 2007.

[10] F. Lopes, F. Delicato, T. Batista, and P. F. Pires, "Context-based Heterogeneous Middleware Integration", the International Workshop on Middleware for Ubiquitous and Persative Systems, pp. 13-19, 2009.

[11] NETL, "Oil and Natural Gas Porjects: Transmission, Distribution, and Refining", http://www.netl.doe.gov, 2005.

[12] J. S. Oh, J. S. Park, and J. R. Kwon, "Selecting the Wireless Communication Methods for Establishing Ubiquitous City-gas Fiaclities in Korea" Springer-Verlag Lecture Notes in Computer Science, vol. 5576, pp. 823-828, 2009.

[13] A. R. Ricardo, E. Markus, and S. S. Thiago, "Middleware for Ubiquitous Context-Awareness", the International Workshop on Middleware for Pervasive and ad-hoc Computing, pp. 43-48, 2008.

[14] C. Srisathapornhat, C. Jaikaeo, and C. Shen, "Sensor Information Networking Architecture and Applications" Journal of IEEE Personal Communiations", vol. 8, no. 4, pp. 52-59, 2004.

[15] H. Wang, Y. Zhang, and J. Cao, "Ubiquitois Computing Environments and Its Usage Access Control", the International Conference on Scalable Information System, pp. 1-10, 2006.

[16] Y. Zhagm, J. Luo, and H. Hu, "Wireless Mesh Networks: Architecture, Protocol, and Standards", Auerbach Publications, 2007. 